\title{
Raman light scattering for systems with strong short-range interaction
}

\author{
I.V.Stasyuk, T.S.Mysakovych \\ Institute for Condensed Matter Physics \\ of the National Academy of Sciences of Ukraine, \\ 1 Svientsitskii Str., 79011 Lviv, Ukraine
}

Received September 29, 1999

\begin{abstract}
Various type contributions to Raman light scattering are investigated for the Hubbard, $t-J$ and pseudospin-electron models. To construct the polarizability operator the microscopic approach is used, which is based on the operator expansion in the terms of the Hubbard operators using $t$ and $J$ as formal parameters of the expansion. Two different contributions to the dipole momentum are taken into account: one is connected with the nonhomeopolarity of filling of the electron states on a site, another - with the dipole transitions from the ground state to the excited ones (for the case of the Hubbard model) and with the dipole momentum of the pseudospins (for the case of the pseudospin- electron model). The general expressions for the scattering tensor components describing the magnon, electron (intraand interband) and pseudospin scattering are obtained. The resonant and nonresonant contributions are separated; their role at the change of the hole concentration due to doping is studied. The dependence of the Raman scattering tensor on the polarization of the incident and scattered light is investigated.
\end{abstract}

Key words: Raman scattering, Hubbard correlation, pseudospin-electron model, polarizability operator

PACS: $72.10 . D p, 72.10 . D i, 74.20$

\section{Introduction}

The problem of nonphonon contributions to Raman light scattering in the crystals with the strong short-range Hubbard-type interaction between electrons remains a subject of interest during the last years in spite of the success achieved in describing the magnetic and electron Raman scattering in the systems with antiferromagnetic ordering [1-3]. The approach used by [1] was based on the techniques of constructing the effective Hamiltonian of interaction between the system and the incident light. It was shown in [1] that the main contribution to Raman scattering in antiferromagnets is due to the two-magnon scattering; the magnons which participate in 
this scattering are the edge magnons of the Brillouin zone. The effective scattering Hamiltonian has the structure similar to the operator of the exchange interaction and is proportional to the scalar product of spin operators on the neighbouring lattice sites; scattering intensity is determined by the square of the parameter of the electron exchange through the excited states of atoms. This idea was partially used in [2] where in the case of Hubbard model the electron scattering contributions, connected with the lower Hubbard subband, were considered. At the half filling $(n=1)$ and at strong correlation $(U \gg t)$ an antiferromagnetic state is the ground one. The transitions with the participation of the upper subband make it possible to achieve the two-magnon scattering similar to that considered in [1]. Besides such kind of scattering that can be referred to the resonant type (the scattering operator is proportional to $t^{2} /\left(U-\hbar \omega_{1}\right)$, the contributions of the higher order with respect to $t /\left(U-\hbar \omega_{1}\right)$ were analyzed. The corresponding components of the scattering operator being projected on the states of the lower Hubbard subband (at the homeopolarity condition $\sum_{\sigma} n_{i \sigma}=1$ ) are expressed in terms of spin operator products; their form depends on the geometry of scattering (i.e the polarization of the incident and the scattered light) $[2,3]$. The attempt was made to also describe the so-called nonresonant contributions to the scattering which manifest themselves in the doped case (at the presence of holes, $n<1)$ and are connected with the intraband electron transitions. There have been separated contributions proportional to $t^{2}$; the conditions at which such terms remain nonzero ones in the limit $\vec{k}_{2}-\vec{k}_{1} \rightarrow 0$ were considered $\left(\vec{k}_{1}\right.$ and $\vec{k}_{2}$ are the wave vectors of the incident and the scattered light, respectively).

The aim of this work is to develop a scheme that will make it possible to make a deeper and a more consistent investigation of the various type nonphonon contributions to the Raman scattering in strongly correlated electron systems. A microscopic approach which was proposed in [4-6] is applied. This method is based on the construction of the polarizability operator $\hat{P}$ of the system by means of a direct solving of the equations of motion with the use of the operator expansion. Within this scheme the electron contributions to the Raman scattering in the Hubbard model are considered, the magnon component of the scattering in the $t-J$ model is separated (at the mean-field type consideration of the doping level) as well as the additional contributions appearing in these models due to the exchange interaction via the excited states are investigated. On the same basis the possible mechanisms of the Raman scattering in systems described by the pseudospin-electron model are studied. This model was proposed in connection with the investigation of locally anharmonic phenomena in high- $\mathrm{T}_{\mathrm{c}}$ superconducting crystals (anharmonic subsystem of ions in the double-minimum potential wells is described by means of pseudospin; the electron subsystem possesses a strong short-range correlation similar to that in the case of Hubbard model $[7,8])$.

We start from the explicit expression for the cross-section of Raman light scattering $([4,5])$ :

$$
\frac{\partial^{2} \sigma}{\partial \Omega \partial \omega_{2}}=\frac{1}{\left(4 \pi \varepsilon_{0}\right)^{2}} \sqrt{\frac{\varepsilon_{1}}{\varepsilon_{2}}} \frac{\omega_{2}^{3} \omega_{1}}{\hbar^{2} c^{4}} \sum_{\alpha \beta \alpha^{\prime} \beta^{\prime}} e_{1 \alpha} e_{2 \beta} e_{1 \alpha^{\prime}} e_{2 \beta^{\prime}} H_{k_{2}, k_{1}}^{\beta^{\prime} \alpha^{\prime}, \beta, \alpha}\left(\omega_{1}, \omega_{2}\right)
$$


here $\vec{e}_{1}, \vec{e}_{2}$ are polarization vectors; $\omega_{1}, \omega_{2}$ are incident and scattered light frequencies; $\varepsilon_{1,2} \equiv \varepsilon\left(\omega_{1}, \omega_{2}\right) ; H_{k_{2}, k_{1}}^{\beta^{\prime} \alpha^{\prime}, \alpha}\left(\omega_{1}, \omega_{2}\right)$ is the Raman scattering tensor:

$$
H_{k_{2},-k_{1} ;-k_{2}, k_{1}}^{\beta^{\prime} \alpha^{\prime}, \beta, \alpha}\left(\omega_{1}, \omega_{2}\right)=\frac{1}{2 \pi} \int_{-\infty}^{+\infty} \mathrm{d} t \mathrm{e}^{\mathrm{i}\left(\omega_{1}-\omega_{2}\right) t}\left\langle\hat{P}_{\vec{k}_{2}-\vec{k}_{1}}^{\beta^{\prime} \alpha^{\prime}}\left(-\omega_{1}, t\right) \hat{P}_{-\vec{k}_{2} \vec{k}_{1}}^{\beta \alpha}\left(\omega_{1}, 0\right)\right\rangle,
$$

where $\hat{P}$ is the polarizability operator

$$
\hat{P}_{\vec{k}^{\prime} \vec{k}}^{\beta \alpha}(\omega, t)=-\int_{-\infty}^{+\infty} \mathrm{d} s \mathrm{e}^{\mathrm{i} \omega(t-s)}\left\{\left\{\hat{M}^{\beta}\left(\overrightarrow{k^{\prime}}, t\right) \mid \hat{M}^{\alpha}(\vec{k}, s)\right\}\right\}
$$

Here $\hat{M}^{\alpha}(\vec{k})$ is a dipole momentum of a crystal unit cell in the $\vec{k}$-representation and the symbol $\left\{\left\{\hat{M}^{\beta}\left(\overrightarrow{k^{\prime}}, t\right) \mid \hat{M}^{\alpha}(\vec{k}, s)\right\}\right\}$ stands for "unaveraged" Green's function defined in the following way $[6]$ :

$$
\left\{\left\{A(t) \mid B\left(t^{\prime}\right)\right\}\right\}=-\mathrm{i} \Theta\left(t-t^{\prime}\right)\left[A(t), B\left(t^{\prime}\right)\right] ;
$$

operators $A(t), B\left(t^{\prime}\right)$ are given in the Heisenberg representation.

The equations of motion for this function have the following form

$$
\begin{aligned}
& \hbar \omega_{1}\{\{A \mid B\}\}_{\omega_{1}, \omega_{2}}=\frac{\hbar}{2 \pi}[A, B]_{\omega_{1}-\omega_{2}}+\{\{[A, H] \mid B\}\}_{\omega_{1}, \omega_{2}}, \\
& \hbar \omega_{2}\{\{A \mid B\}\}_{\omega_{1}, \omega_{2}}=\frac{\hbar}{2 \pi}[A, B]_{\omega_{1}-\omega_{2}}-\{\{A \mid[B, H]\}\}_{\omega_{1}, \omega_{2}},
\end{aligned}
$$

where corresponding Fourier transforms are introduced. The equations (5), (6) are applied to construct the polarizability operator; the solutions of these equations are built in the form of operator series in powers of certain parameters of Hamiltonian $H$. In this work in the case of the models of the Hubbard model type the expansion in terms of the electron transfer parameter $t$ is used.

\section{The Hubbard model}

First let us consider the case of the Hubbard model

$$
\hat{H}=\sum_{i, j} t_{i, j} \hat{c}_{i, \sigma}^{\dagger} \hat{c}_{j, \sigma}+U \sum_{i} \hat{n}_{i, \uparrow} \hat{n}_{i, \downarrow}-\sum_{i} \mu \hat{n}_{i}
$$

where the first and the second terms describe the electron transfer and short-range electron correlation, respectively; $\mu$ is chemical potential. We will restrict ourselves to the case of large $U$, so we can make expansion in powers of $t$ at the construction of the $\hat{P}$-operator, considering only the terms which are linear and quadratic in $t$. In the case of the Hubbard model the dipole momentum of a unit cell has a form

$$
\hat{\vec{M}}_{i}=e \vec{R}_{i}\left(\hat{n}_{i, \uparrow}+\hat{n}_{i, \downarrow}\right) .
$$


In this expression the nonhomeopolarity of filling of the electron states on lattice sites is taken into account. It is useful to consider the following single-site basis of the states $\left|n_{i, \downarrow}, n_{i, \uparrow}\right\rangle$

$$
|1\rangle=|0,0\rangle, \quad|2\rangle=|1,1\rangle, \quad|3\rangle=|1,0\rangle, \quad|4\rangle=|0,1\rangle
$$

and to introduce the Hubbard operators $X^{r, s}=|r\rangle\langle s|$.

To calculate Green's function $\left\{\left\{\hat{M}_{k}^{\alpha} \mid \hat{M}_{l}^{\beta}\right\}\right\}$ we first use the equation of motion (5):

$$
\left\{\left\{\hat{M}_{k}^{\alpha} \mid \hat{M}_{l}^{\beta}\right\}\right\}=\frac{e R_{k}^{\alpha}}{\hbar \omega_{1}} \sum_{i, j, \sigma} t_{i, j}\left(\delta_{i, k}-\delta_{j, k}\right)\left\{\left\{\hat{c}_{i, \sigma}^{\dagger} \hat{c}_{j, \sigma} \mid \hat{M}_{l}^{\beta}\right\}\right\}
$$

Then we use the equation of motion written in the form (6):

$$
\begin{aligned}
\left\{\left\{\hat{c}_{i, \sigma}^{\dagger} \hat{c}_{j, \sigma} \mid \hat{M}_{l}^{\beta}\right\}\right\}= & {\left[\frac{\hbar}{2 \pi}\left(\delta_{j, l}-\delta_{i, l}\right) \hat{c}_{i, \sigma}^{\dagger} \hat{c}_{j, \sigma}\right.} \\
& \left.-\sum_{s, p \sigma^{\prime}} t_{s, p}\left(\delta_{s, l}-\delta_{p, l}\right)\left\{\left\{\hat{c}_{i, \sigma}^{\dagger} \hat{c}_{j, \sigma} \mid \hat{c}_{s, \sigma^{\prime}}^{\dagger} \hat{c}_{p, \sigma^{\prime}}\right\}\right\}\right] \frac{e R_{l}^{\beta}}{\hbar \omega_{2}}
\end{aligned}
$$

To calculate the function $\left\{\left\{\hat{c}_{i, \sigma}^{\dagger} \hat{c}_{j, \sigma} \mid \hat{c}_{s, \sigma^{\prime}}^{\dagger} \hat{c}_{p, \sigma^{\prime}}\right\}\right\}$ we again write the equation of motion (5) and neglect the terms which are proportional to $t$. Using this scheme, the following expression for Green's function $\left\{\left\{\hat{M}_{k}^{\alpha} \mid \hat{M}_{l}^{\beta}\right\}\right\}$ is obtained up to the terms of the second order of $t$ :

$$
\begin{aligned}
\left\{\left\{\hat{M}_{k}^{\alpha} \mid \hat{M}_{l}^{\beta}\right\}\right\}= & {\left[\sum_{i, j, \sigma} t_{i, j}\left(\delta_{i, k}-\delta_{j, k}\right)\left(\delta_{j, l}-\delta_{i, l}\right) \hat{c}_{i, \sigma}^{\dagger} \hat{c}_{j, \sigma}\right.} \\
& \left.-\frac{1}{\hbar \omega_{1}+U} \hat{A}_{k l}^{\dagger}+\frac{1}{\hbar \omega_{1}-U} \hat{A}_{k l}+\frac{1}{\hbar \omega_{1}} \hat{B}_{k l}\right] \frac{e^{2} R_{k}^{\alpha} R_{l}^{\beta}}{2 \pi \hbar \omega_{1} \omega_{2}},
\end{aligned}
$$

here $U$ plays the role of the energetic distance between the two levels which are responsible for scattering,

$$
\begin{aligned}
& \hat{A}_{k l}=\sum_{i, j, s} t_{i, j} t_{s, j}\left(\delta_{i, k}-\delta_{j, k}\right)\left(\delta_{j, l}-\delta_{s, l}\right) \\
& \times {\left[X_{i}^{31}\left(X_{j}^{22}+X_{j}^{44}\right)\left(X_{\mathrm{s}}^{13}-X_{\mathrm{s}}^{42}\right)+X_{i}^{41}\left(X_{\mathrm{s}}^{31}-X_{\mathrm{s}}^{24}\right) X_{j}^{12}\right.} \\
&-\left(X_{\mathrm{s}}^{41}+X_{\mathrm{s}}^{23}\right)\left(X_{j}^{11}+X_{j}^{44}\right) X_{i}^{32}+X_{i}^{41} X_{j}^{34}\left(X_{\mathrm{s}}^{42}-X_{\mathrm{s}}^{13}\right) \\
&+X_{i}^{41}\left(X_{j}^{33}+X_{j}^{22}\right)\left(X_{\mathrm{s}}^{32}+X_{\mathrm{s}}^{14}\right)+X_{j}^{21}\left(X_{\mathrm{s}}^{13}-X_{\mathrm{s}}^{42}\right) X_{i}^{32} \\
&-\left(X_{\mathrm{s}}^{31}-X_{\mathrm{s}}^{24}\right) X_{j}^{43} X_{i}^{32}-X_{i}^{31} X_{j}^{43}\left(X_{\mathrm{s}}^{14}+X_{\mathrm{s}}^{32}\right) \\
&-X_{i}^{31}\left(X_{\mathrm{s}}^{41}+X_{\mathrm{s}}^{23}\right) X_{j}^{12} X_{j}^{21}\left(X_{\mathrm{s}}^{14}+X_{\mathrm{s}}^{32}\right) X_{i}^{42}+ \\
&\left.+\left(X_{\mathrm{s}}^{41}+X_{\mathrm{s}}^{23}\right) X_{j}^{34} X_{i}^{42}+\left(X_{\mathrm{s}}^{31}-X_{\mathrm{s}}^{24}\right)\left(X_{j}^{11}+X_{j}^{33}\right) X_{i}^{42}\right], \\
& \hat{B}_{k l}=\left(\hat{A}_{k l}^{\dagger}+\hat{A}_{k l}\right)\left(\begin{array}{c}
X^{11} \rightarrow X^{22} \\
X^{34}, X^{43} \rightarrow-X^{34},-X^{43}
\end{array}\right) .
\end{aligned}
$$


Comparing with the results obtained in $[2,3]$, we can see that the second order terms in our expression, when $i=s$ and $n_{i, \uparrow}+n_{i, \downarrow}=1$, are the same as the scattering Hamiltonian obtained in [2]:

$$
\left\{\left\{\hat{M}_{k}^{\alpha} \mid \hat{M}_{l}^{\beta}\right\}\right\} \sim \sum_{i, j}\left(\delta_{i, k}-\delta_{j, k}\right)\left(\delta_{i, l}-\delta_{j, l}\right) \frac{t_{i, j}^{2}}{\hbar \omega_{1}-U} \hat{\vec{S}}_{i} \hat{\vec{S}}_{j}
$$

(considering only the resonant term $\left.\sim\left(\hbar \omega_{1}-U\right)^{-1}\right)$. Such a structure of the scattering Hamiltonian leads to the magnon Raman scattering. However, there are some additional terms in (12) which were not presented in [2]. These terms are connected with electron transitions between the next nearest neighbours and are responsible for the scattering with the participation of holes or extra electrons, which is actual in the doped case $(n \neq 1)[9]$.

Now let us calculate the scattering tensor. To deal with the definition (2) we will use the formula:

$$
\left\langle\hat{C}\left(\omega_{1}-\omega_{2}\right) \hat{D}\left(\omega_{1}^{\prime}-\omega_{1}\right)\right\rangle=\delta\left(\omega_{1}^{\prime}-\omega_{2}\right) \frac{2 \Im \mathrm{m}\langle\langle\hat{D} \mid \hat{C}\rangle\rangle_{\omega+\mathrm{i} \varepsilon}}{\mathrm{e}^{\beta \hbar \omega}-1}{ }_{\omega=\omega_{2}-\omega_{1}} .
$$

In consequence we obtain the following expression for the scattering tensor:

$$
\begin{aligned}
H_{k_{2}, k_{1}}^{\beta^{\prime} \alpha^{\prime}, \beta, \alpha}\left(\omega_{1}, \omega_{2}\right)= & \sum_{\substack{n, n^{\prime}, n_{1}, n_{1}^{\prime} \\
i, j, i_{1}, n_{1}}} \mathrm{e}^{\mathrm{i}\left(\vec{k}_{2}\left(\vec{R}_{n i}-\vec{R}_{n_{1} i_{1}}\right)-\vec{k}_{1}\left(\vec{R}_{n^{\prime} j}-\vec{R}_{n_{1}^{\prime} j_{1}}\right)\right)} \\
& \times \frac{e^{4} 2 \Im \mathrm{m}\left\langle\left\langle\hat{A} \mid \hat{A}^{\dagger}\right\rangle\right\rangle_{\omega=\omega_{2}-\omega_{1}} R_{n i}^{\alpha} R_{n^{\prime} j}^{\beta} R_{n_{1} i_{1}}^{\alpha^{\prime}} R_{n_{1}^{\prime} j_{1}}^{\beta^{\prime}}}{\left(\mathrm{e}^{\beta \hbar \omega}-1\right) \hbar \omega_{1} \omega_{2}\left(\hbar \omega_{1}-U\right)^{2}},
\end{aligned}
$$

here only the resonant term $\left(\hbar \omega_{1} \sim U\right)$ is retained. To calculate Green's function $\left\langle\left\langle\hat{A} \mid \hat{A}^{\dagger}\right\rangle\right\rangle$ using the Hamiltonian of the Hubbard model, we will use a decoupling procedure for Fermi type operators:

$$
\left\langle X^{p q}(t) X^{r s}(t) X^{m n} X^{p l}\right\rangle \approx\left\langle X^{p q}(t) X^{p l}\right\rangle\left\langle X^{r s}(t) X^{m n}\right\rangle \pm\left\langle X^{p q}(t) X^{m n}\right\rangle\left\langle X^{r s}(t) X^{p l}\right\rangle,
$$

having split Boson operators $X^{p p}$ in the product of two Fermi operators: $X^{p p}=$ $X^{p 1} X^{1 p}$. When calculating Green's function $\left\langle\left\langle X^{p q} \mid X^{r s}\right\rangle\right\rangle$ (where $X^{p q}, X^{r s}$ are Fermi operators), we use the simplified version of the Hubbard-I approximation, corresponding to the case of independent subbands, and restrict ourselves to the lower Hubbard subband, neglecting contributions connected with the state $|2\rangle$. After some algebra we obtain the following result:

$$
\begin{aligned}
& H_{k_{2}, k_{1}}^{\beta^{\prime} \alpha^{\prime}, \beta, \alpha}\left(\omega_{1}, \omega_{2}\right)=\frac{32 e^{4} a^{4} t^{4}\left\langle X^{11}+X^{33}\right\rangle^{4}}{N^{2} \omega_{1} \omega_{2}\left(\hbar \omega_{1}-U\right)^{2}} \\
& \times\left\{2 \sum_{q_{1}, q_{2}, q_{3}, q} \frac{\delta\left(k_{2}-k_{1}-q_{1}+q+q_{2}-q_{3}\right)}{\left(\mathrm{e}^{\beta\left(\mu-\left\langle X^{11}+X^{33}\right\rangle t(q)\right)}+1\right)\left(\mathrm{e}^{-\beta\left(\mu-\left\langle X^{11}+X^{33}\right\rangle t\left(q_{1}\right)\right)}+1\right)}\right. \\
& \times \frac{\delta\left(\omega-\frac{\left\langle X^{11}+X^{33}\right\rangle}{\hbar}\left(t\left(q_{1}\right)-t(q)+t\left(q_{3}\right)-t\left(q_{2}\right)\right)\right)}{\left(\mathrm{e}^{\beta\left(\mu-\left\langle X^{11}+X^{33}\right\rangle t\left(q_{2}\right)\right)}+1\right)\left(\mathrm{e}^{-\beta\left(\mu-\left\langle X^{11}+X^{33}\right\rangle t\left(q_{3}\right)\right)}+1\right)}
\end{aligned}
$$




$$
\begin{aligned}
& \times \sin \left(q^{\alpha^{\prime}}\right)\left(\sin \left(q_{1}^{\beta}\right)+\sin \left(q_{3}^{\beta}\right)\right) \sin \left(q_{1}^{\beta^{\prime}}\right)\left(\sin \left(q^{\alpha}\right)+\sin \left(q_{1}^{\alpha}\right)\right) \\
& +\sum_{q_{1}, q_{2}, q_{3}, q} \frac{\delta\left(k_{2}-k_{1}-q_{1}+q\right)}{\left(\mathrm{e}^{\beta\left(\mu-\left\langle X^{11}+X^{33}\right\rangle t(q)\right)}+1\right)\left(\mathrm{e}^{-\beta\left(\mu-\left\langle X^{11}+X^{33}\right\rangle t\left(q_{1}\right)\right)}+1\right)} \\
& \times \frac{\delta\left(\omega-\frac{\left\langle X^{11}+X^{33}\right\rangle}{\hbar}\left(t\left(q_{1}\right)-t(q)\right)\right)}{\left(\mathrm{e}^{-\beta\left(\mu-\left\langle X^{11}+X^{33}\right\rangle t\left(q_{2}\right)\right)}+1\right)\left(\mathrm{e}^{-\beta\left(\mu-\left\langle X^{11}+X^{33}\right\rangle t\left(q_{3}\right)\right)}+1\right)} \\
& \times\left(\operatorname { s i n } ( q ^ { \alpha } ) \left(\sin \left(q_{1}^{\beta}\right)+\left(\sin \left(q_{3}^{\alpha}\right) \sin \left(q_{3}^{\beta}\right)\right)\left(\sin \left(q_{2}^{\alpha^{\prime}}\right) \sin \left(q_{2}^{\beta^{\prime}}\right)+\left(\sin \left(q^{\alpha^{\prime}}\right) \sin \left(q_{1}^{\beta^{\prime}}\right)\right)\right\}\right.\right.
\end{aligned}
$$

here $a$ is a lattice constant. The average $\left\langle X^{11}+X^{33}\right\rangle$ is determined in a self-consistent way $[7,9]$. From the expression (18) we can see that the difference between the frequencies of the scattered and the incident light waves is connected with the twoelectron transitions in the band:

$$
\begin{aligned}
& \omega=\omega_{2}-\omega_{1}=\frac{\left\langle X^{11}+X^{33}\right\rangle}{\hbar}\left[t\left(q_{1}\right)+t\left(q_{3}\right)-t\left(q_{2}\right)-t\left(q_{3}\right)\right], \\
& q_{1}+q_{3}=q_{2}+q+k_{2}-k_{1}
\end{aligned}
$$

as well as with the one-electron intraband transitions:

$$
\omega_{2}-\omega_{1}=\frac{\left\langle X^{11}+X^{33}\right\rangle}{\hbar}\left[t\left(q_{1}\right)-t(q)\right], \quad q_{1}=q+k_{2}-k_{1} .
$$

Similar one-electron transitions can also be obtained from the nonresonant term in (12), which is linear in $t$. The last contribution to the scattering tensor was considered in $[2,3]$ in the case of hole doping $(n<1)$. One can see that in the simple single-band case the frequency change (20) tends to zero due to the inequality $\left|k_{2}-k_{1}\right| \ll q, q_{1}$ (that is characteristic of light wave vectors). By the same reason the corresponding term in (18) vanishes in the $T=0$ limit.

Calculating Green's function, we used the Hubbard Hamiltonian with the excluded state $|2\rangle$, so we have obtained only the electron (hole) scattering. To consider the magnon scattering we will deal with the $t-J$ model.

\section{The extended $t-J$ model}

In the case of nearly half filling $\left\langle n_{i, \uparrow}+n_{i, \downarrow}\right\rangle \approx 1$ and $U \gg t$ the Hubbard Hamiltonian can be reduced to the effective Hamiltonian of the so-called $t-J$ model:

$$
\hat{H}_{t-J}=\sum_{i, j, \sigma} t_{i, j} \hat{\widetilde{c}}_{j, \sigma}^{\dagger} \hat{\widetilde{c}}_{i, \sigma}+\sum_{i, j} J_{i, j}\left(\hat{\vec{S}}_{i} \hat{\vec{S}}_{j}-\frac{\hat{n}_{i, \uparrow} \hat{n}_{j, \downarrow}}{4}\right)-\sum_{i} \mu \hat{n}_{i}
$$

here $\hat{\widetilde{c}}_{i, \sigma}=\hat{c}_{i, \sigma}\left(1-\hat{n}_{i,-\sigma}\right), J_{i, j}=4 t_{i, j}^{2} / U$ is the constant of the antiferomagnetic type exchange interaction. Let us extend this model taking into account the excited atomic states $\varphi_{\mathrm{exc}}^{\alpha}$, having different parity with respect to the ground state $\varphi_{0}$ as 
well as their exchange and Coulomb interaction with the ground $\operatorname{state}^{1}[1]$ :

$$
\begin{aligned}
\hat{H} & =\hat{H}_{t-J}+(E-\mu) \sum_{i, \sigma, \alpha} \hat{a}_{\alpha i, \sigma}^{\dagger} \hat{a}_{\alpha i, \sigma}+\sum_{i, j, \sigma, \alpha, \beta} M_{i, j}^{\alpha, \beta}\left(X_{i}^{31} X_{j}^{13}+X_{i}^{41} X_{j}^{14}\right) \hat{a}_{\alpha, j, \sigma}^{\dagger} \hat{a}_{\beta, i, \sigma}^{\dagger} \\
& -\sum_{i, j, \alpha, \beta} K_{i, j}^{\alpha, \beta}\left(X_{i}^{33} \hat{a}_{\alpha j, \downarrow}^{\dagger} \hat{a}_{\beta j, \downarrow}+X_{i}^{44} \hat{a}_{\alpha j, \uparrow}^{\dagger} \hat{a}_{\beta j, \uparrow}+X_{i}^{34} \hat{a}_{\alpha j, \uparrow}^{\dagger} \hat{a}_{\beta j, \downarrow}+X_{i}^{43} \hat{a}_{\alpha j, \downarrow}^{\dagger} \hat{a}_{\beta j, \uparrow}\right),(22)
\end{aligned}
$$

the indices $\alpha, \beta$ refer to the orbitally degenerated excited states; $\mathrm{E}$ is the excitation energy. $K_{i, j}^{\alpha, \beta}, M_{i, j}^{\alpha, \beta}$ are additional interaction constants. The Hamiltonian is written in terms of the Hubbard operators $X^{r s}$. The relation between the spin operator $\vec{S}_{i}$ and the operators $X^{r s}$ is as follows:

$$
S_{i}^{z}=\frac{1}{2}\left(X_{i}^{44}-X_{i}^{33}\right), \quad S^{+}=S_{i}^{x}+\mathrm{i} S_{i}^{y}=X_{i}^{43}, \quad S^{-}=S_{i}^{x}-\mathrm{i} S_{i}^{y}=X_{i}^{34} .
$$

Let us take the dipole momentum in the form:

$$
\hat{M}_{i}^{\alpha}=d^{\alpha}\left(\hat{a}_{\alpha, i, \downarrow}^{\dagger} X_{i}^{13}+\hat{a}_{\alpha, i, \uparrow}^{\dagger} X^{14}+\text { h.c. }\right)
$$

separating the component, which is connected with the dipole transitions between the ground and the excited states; $d^{\alpha}=\left\langle\varphi_{0}\left|r^{\alpha}\right| \varphi_{\text {exc }}^{\alpha}\right\rangle$. We make expansion in terms of $t / E, M / E, K / E$ at the construction of the polarizability operator, considering the linear terms in $M / E, K / E$ and quadratic in $t / E$. Using the method described in the previous section the following formula for Green's function $\left\{\left\{\hat{M}_{k}^{\alpha} \mid \hat{M}_{l}^{\beta}\right\}\right\}$ is obtained:

$$
\begin{aligned}
& \left\{\left\{\hat{M}_{k}^{\alpha} \mid \hat{M}_{l}^{\beta}\right\}\right\}=\frac{\hbar e^{2} d^{\alpha} d^{\beta}}{2 \pi\left(\hbar \omega_{1}-E\right)\left(\hbar \omega_{2}-E\right)} \\
& \quad \times\left[-\sum_{i} \delta_{k, l} \delta_{\alpha, \beta} t_{i, k}\left(X_{i}^{31} X_{k}^{13}+X_{i}^{41} X_{k}^{14}\right)-\sum_{i} \delta_{k, l} 2 J_{i, k}\left(\hat{\vec{S}}_{i} \hat{\vec{S}}_{k}-\frac{\hat{n}_{i} \hat{n}_{k}}{4}\right) \delta_{\alpha, \beta}\right. \\
& \left.\quad-M_{l, k}^{\alpha \beta}\left(\hat{\vec{S}}_{l} \hat{\vec{S}}_{k}+\frac{\hat{n}_{l} \hat{n}_{k}}{4}\right)-\sum_{i} \delta_{l, k} K_{i, k}^{\alpha \beta}\left(\hat{\vec{S}}_{i} \hat{\vec{S}}_{k}+\frac{\hat{n}_{i} \hat{n}_{k}}{4}\right)\right] \\
& \quad+\frac{\hbar e^{2} d^{\alpha} d^{\beta} \delta_{k, l} \delta_{\alpha, \beta}}{2 \pi\left(\hbar \omega_{1}-E\right)^{2}\left(\hbar \omega_{2}-E\right)} \sum_{i, j} t_{i, k} t_{j, k}\left(X_{i}^{31} X_{j}^{13}+X_{i}^{41} X_{j}^{14}\right) .
\end{aligned}
$$

Here we include only the resonant terms and leave out the terms which concern the excited states.

The spin part of the expression for $\left\{\left\{\hat{M}_{k}^{\alpha} \mid \hat{M}_{l}^{\beta}\right\}\right\}$ is similar to that obtained in [1] for antiferromagnet. The formula transforms into the simple product of spin operators $\hat{\vec{S}}_{i} \hat{\vec{S}}_{j}$ in the case of homeopolarity: $n_{i, \uparrow}+n_{i, \downarrow}=1$ (when the hole doping level is equal to zero) and the terms which are linear and quadratic in $t / E$ arise from the pure band transitions.

Let us pass to the scattering tensor. A spin-polaron approach can be used for the $t-J$ model in the region of small hole concentrations [10,11]. We introduce for the

\footnotetext{
${ }^{1}$ We take into account the possibility of the orbital degeneration of the excited state. In the case of $p$-like function $\varphi_{\mathrm{exc}}^{\alpha}$ and the quasi-two-dimensional structure $\alpha=x, y$ considered below.
} 
electron operators $\hat{\widetilde{c}}_{i, \sigma}$ the following representation in the sublattices with spin up (i $\in \uparrow)$ and spin down $(i \in \downarrow)$ ground states (that correspond to the antiferromagnetic ordering)

$$
\hat{\widetilde{c}}_{i, \uparrow}=\hat{h}_{i 1}^{\dagger}, \quad \hat{\widetilde{c}}_{i, \downarrow}=\hat{h}_{i 1}^{\dagger} \hat{b}_{i_{1}}(i \in \uparrow) ; \quad \hat{\widetilde{c}}_{i, \downarrow}=\hat{h}_{i 2}^{\dagger}, \quad \hat{\widetilde{c}}_{i, \uparrow}=\hat{h}_{i 2}^{\dagger} \hat{b}_{i 2}(i \in \downarrow),
$$

here $\hat{h}_{i 1}$ and $\hat{h}_{i 2}$ are hole spinless operators and $\hat{b}_{i 1}, \hat{b}_{i 2}$ are magnon operators on two sublattices. Performing the canonical transformation for Fourier components

$$
b_{k 1}=v_{k} \alpha_{k}+u_{k} \beta_{-k}^{+}, \quad b_{k 2}=v_{k} \beta_{k}+u_{k} \alpha_{-k}^{+},
$$

we obtain the following Hamiltonian of the spin-polaron model:

$$
\begin{aligned}
H_{t-J}= & \sum_{k, q}\left(h_{k 1}^{+} h_{k-q 2}\left[g(k, q) \alpha_{q}+g(q-k, q) \beta_{q}^{+}\right]+\text {h.c. }\right) \\
& -\mu \sum_{k}\left(h_{k 1}^{+} h_{k 1}+h_{k 2}^{+} h_{k 2}\right)+\sum_{q} \omega_{q}\left(\alpha_{q}^{+} \alpha_{q}+\beta_{q}^{+} \beta_{q}\right) .
\end{aligned}
$$

Here

$$
\begin{aligned}
& g(k, q)=\frac{4 t}{\sqrt{N / 2}}\left(u_{q} \gamma_{k-q}+v_{q} \gamma_{k}\right), \quad u_{k}=\sqrt{\frac{1+\nu_{k}}{2 \nu_{k}}}, \\
& v_{k}=-\operatorname{sign}\left(\gamma_{k}\right) \sqrt{\frac{1-\nu_{k}}{2 \nu_{k}}}, \quad \nu_{k}=\sqrt{1-\gamma_{k}^{2}}, \\
& \gamma_{k}=\frac{1}{4} \sum_{r} \mathrm{e}^{\mathrm{i} k r}, \quad \omega_{k}=2 J(1-2 \delta)^{2} \nu_{k}, \quad 2 \delta=\left\langle h_{i 1}^{+} h_{i 1}\right\rangle+\left\langle h_{i 2}^{+} h_{i 2}\right\rangle .
\end{aligned}
$$

This representation excludes doubly occupied states and takes into account strong antiferromagnetic spin correlations at the electron hopping.

Now we rewrite the Raman scattering tensor in terms of the hole spinless operators and the magnon operators:

$$
\begin{aligned}
H_{k_{1}, k_{2}}^{\beta^{\prime} \alpha^{\prime}, \beta \alpha}\left(\omega_{1}, \omega_{2}\right)= & \sum_{\substack{n, n^{\prime}, n_{1}, n^{\prime} 1 \\
i, i_{1}, j, j_{1}}} \mathrm{e}^{\mathrm{i}\left(\vec{k}_{2}\left(\vec{R}_{n i}-\vec{R}_{n_{1} i_{1}}\right)-\vec{k}_{1}\left(\vec{R}_{n^{\prime} j}-\vec{R}_{n_{1}^{\prime} j_{1}}\right)\right)} \\
& \times \frac{e^{4} \hbar^{2} d^{\alpha} d^{\alpha^{\prime}} d^{\beta} d^{\beta^{\prime}} 2 \Im \mathrm{m}\left\langle\left\langle T_{n_{1} i_{1}, n_{1}^{\prime} j_{1}}^{\alpha \beta} \mid T_{n i, n^{\prime} j}^{\alpha^{\prime} \beta^{\prime}}\right\rangle\right\rangle}{\left(\mathrm{e}^{\beta \hbar \omega}-1\right)\left(\hbar \omega_{1}-E\right)^{2}\left(\hbar \omega_{2}-E\right)^{2}},
\end{aligned}
$$

here the operator $T_{n_{1} i_{1}, n_{1}^{\prime} j_{1}}^{\alpha \beta}$ has the form:

$$
\begin{aligned}
& T_{n_{1} i_{1}, n_{1}^{\prime} j_{1}}^{\alpha \beta}= \\
& \quad=\left[\delta_{\alpha \beta} \delta_{n_{1} n_{1}^{\prime}} \delta_{i_{1} j_{1}} \sum_{m, l} 2 J_{m l, n_{1} i_{1}}\left(b_{m l} b_{n_{1} i_{1}}+b_{m l}^{+} b_{n_{1} i_{1}}^{+}-n_{m l} n_{n_{1} i_{1}}-1\right) h_{m l} h_{m l}^{+} h_{n_{1} i_{1}} h_{n_{1} i_{1}}^{+}\right. \\
& \quad+M_{n_{1} i_{1}, n_{1}^{\prime} j_{1}}^{\alpha \beta}\left(b_{n_{1} i_{1}} b_{n_{1}^{\prime} j_{1}}+b_{n_{1}^{\prime} j_{1}}^{+} b_{n_{1} i_{1}}^{+}+n_{n_{1} i_{1}}+n_{n_{1}^{\prime} j_{1}}\right) h_{n_{1} i_{1} i_{1}} h_{n_{1} i_{1}}^{+} h_{n_{1}^{\prime} j_{1}} h_{n_{1}^{\prime} j_{1}}^{+} \\
& \left.\quad+\sum_{m, l} K_{n_{1} i_{1}, m l}^{\alpha \beta}\left(b_{n_{1} i_{1}} b_{m l}+b_{m l}^{+} b_{n_{1} i_{1}}^{+}+n_{n_{1} i_{1}}+n_{m l}\right) h_{m l} h_{m l}^{+} h_{n_{1} i_{1}} h_{n_{1} i_{1}}^{+}\right](1-\delta)^{2} .
\end{aligned}
$$


We will consider the low values of magnon concentration and will not include the term $n_{m l} n_{n_{1} i_{1}}$; we will also use the approximation: $h_{m i} h_{m i}^{+} \rightarrow(1-\delta)$. In this case

$$
\begin{aligned}
T_{n_{1} i_{1}, n_{1}^{\prime} j_{1}}^{\alpha \beta}= & {\left[\delta_{\alpha \beta} \delta_{n_{1} n_{1}^{\prime}} \delta_{i_{1} j_{1}} \sum_{m, l} 2 J_{m l, n_{1} i_{1}}\left(b_{m l} b_{n_{1} i_{1}}+b_{m l}^{+} b_{n_{1} i_{1}}^{+}\right)\right.} \\
& +M_{n_{1} i_{1}, n_{1}^{\prime} j_{1}}^{\alpha \beta}\left(b_{n_{1} i_{1}} b_{n_{1}^{\prime} j_{1}}+b_{n_{1}^{\prime} j_{1}}^{+} b_{n_{1} i_{1}}^{+}+n_{n_{1} i_{1}}+n_{n_{1}^{\prime} j_{1}}\right) \\
& \left.+\sum_{m, l} K_{n_{1} i_{1}, m l}^{\alpha \beta}\left(b_{n_{1} i_{1}} b_{m l}+b_{m l}^{+} b_{n_{1} i_{1}}^{+}+n_{n_{1} i_{1}}+n_{m l}\right)\right](1-\delta)^{2} .
\end{aligned}
$$

So we have to find Green's function built on the magnon operators. In the case when the hole-magnon scattering is not taken into account it can be done using the diagonal part of the magnon Hamiltonian. For instance, let us calculate Green's function $\left\langle\left\langle b_{q 1} b_{q_{2} 2} \mid b_{q_{3} 1}^{+} b_{q_{4} 2}^{+}\right\rangle\right\rangle$(here the magnon operators $b_{q i}, b_{q i}^{+}$are Fourier components of $b_{n i}, b_{n i}^{+}$, respectively). Using the above mentioned canonical transformation, we can write

$$
\begin{aligned}
& \left\langle\left\langle b_{q 1} b_{q_{2}} \mid b_{q_{3} 1}^{+} b_{q_{4} 2}^{+}\right\rangle\right\rangle= \\
& =\left\langle\left\langle\left\langle\alpha_{q_{1}} \alpha_{-q_{2}}^{+} \mid \alpha_{q_{3}}^{+} \alpha_{-q_{4}}\right\rangle\right\rangle u_{q_{1}} u_{q_{3}} v_{q_{2}} v_{q_{4}}+\left\langle\left\langle\beta_{-q_{1}}^{+} \beta_{q_{2}} \mid \beta_{-q_{3}} \beta_{q_{4}}^{+}\right\rangle\right\rangle u_{q_{2}} u_{q_{4}} v_{q_{1}} v_{q_{3}}\right. \\
& \quad+\left\langle\left\langle\alpha_{q_{1}} \beta_{q_{2}} \mid \alpha_{q_{3}}^{+} \beta_{q_{4}}^{+}\right\rangle\right\rangle u_{q_{1}} u_{q_{3}} u_{q_{2}} u_{q_{4}}+\left\langle\left\langle\beta_{-q_{1}}^{+} \alpha_{-q_{2}} \mid \beta_{-q_{3}} \alpha_{-q_{4}}\right\rangle\right\rangle v_{q_{1}} v_{q_{3}} v_{q_{2}} v_{q_{4}} .
\end{aligned}
$$

The functions obtained can be easily calculated using the standard technique of the equations of motion:

$$
\begin{aligned}
\left\langle\left\langle\alpha_{q_{1}} \alpha_{-q_{2}}^{+} \mid \alpha_{q_{3}}^{+} \alpha_{-q_{4}}\right\rangle\right\rangle & =\frac{\hbar}{2 \pi} \delta_{q_{1} q_{3}} \delta_{q_{2} q_{4}} \frac{\left\langle n_{q_{2}}-n_{q_{1}}\right\rangle}{\hbar \omega-\omega_{q_{1}}+\omega_{q_{2}}}, \\
\left\langle\left\langle\beta_{-q_{1}}^{+} \beta_{q_{2}} \mid \beta_{-q_{3}} \beta_{q_{4}}^{+}\right\rangle\right\rangle & =\frac{\hbar}{2 \pi} \delta_{q_{1} q_{3}} \delta_{q_{2} q_{4}} \frac{\left\langle n_{q_{1}}-n_{q_{2}}\right\rangle}{\hbar \omega+\omega_{q_{1}}-\omega_{q_{2}}}, \\
\left\langle\left\langle\alpha_{q_{1}} \beta_{q_{2}} \mid \alpha_{q_{3}}^{+} \beta_{q_{4}}^{+}\right\rangle\right\rangle & =\frac{\hbar}{2 \pi} \delta_{q_{1} q_{3}} \delta_{q_{2} q_{4}} \frac{\left\langle n_{q_{2}}+1+n_{q_{1}}\right\rangle}{\hbar \omega-\omega_{q_{1}}-\omega_{q_{2}}} \\
\left\langle\left\langle\beta_{-q_{1}}^{+} \alpha_{-q_{2}}^{+} \mid \beta_{-q_{3}} \alpha_{-q_{4}}\right\rangle\right\rangle & =-\frac{\hbar}{2 \pi} \delta_{q_{1} q_{3}} \delta_{q_{2} q_{4}} \frac{\left\langle n_{q_{2}}+1+n_{q_{1}}\right\rangle}{\hbar \omega+\omega_{q_{1}}+\omega_{q_{2}}}
\end{aligned}
$$

Similar to this procedure we can find all other functions.

As a result, considering a two-dimensional volume centred square lattice, we can write the diagonal components of the scattering tensor as follows:

$$
\begin{aligned}
& H^{x x ; y y}\left(\omega_{1}, \omega_{2}\right)=\frac{(1-\delta)^{4} 2 \pi e^{4} \hbar^{2} d^{x 4}}{\left(\mathrm{e}^{\beta \hbar \omega}-1\right)\left(\hbar \omega_{1}-E\right)^{2}\left(\hbar \omega_{2}-E\right)^{2}} \\
& \times\left[\sum_{k} 64\left(v_{k}^{2}+u_{k}^{2}\right)^{2} \delta\left(\omega-2 \omega_{k}\right) \cos ^{2}\left(k_{y} / 2\right) \cos ^{2}\left(k_{x} / 2\right)\left(M^{x x}+J+K^{x x}\right)^{2}\left(2 n_{k}+1\right)\right. \\
& \left.\quad+\sum_{k} 4 v_{k}^{2} u_{k}^{2} \delta\left(\omega-2 \omega_{k}\right)\left(M^{x x}+K^{x x}\right)^{2}\left(2 n_{k}+1\right)\right]
\end{aligned}
$$

(here the polarization directions coincide with the crystallographic axes). Here we have put the wave vectors $\vec{k}_{2}, \vec{k}_{1}$ equal to zero because they are small in comparison 
with the edge vector of the Brillouin zone, which plays the most significant role in the scattering. The nondiagonal components of the tensor have the form

$$
\begin{aligned}
& H^{x y ; x y}\left(\omega_{1}, \omega_{2}\right)=\frac{(1-\delta)^{4} 2 \pi \mathrm{e}^{4} \hbar^{2} d^{x 4}}{\left(\mathrm{e}^{\beta \hbar \omega}-1\right)\left(\hbar \omega_{1}-E\right)^{2}\left(\hbar \omega_{2}-E\right)^{2}} \\
& \times\left[\sum_{k} 64\left(v_{k}^{2}+u_{k}^{2}\right)^{2} \delta\left(\omega-2 \omega_{k}\right) \sin ^{2}\left(k_{y} / 2\right) \sin ^{2}\left(k_{x} / 2\right)\left(M^{x y}+K^{x y}\right)^{2}\left(2 n_{k}+1\right)\right] .(
\end{aligned}
$$

The main contribution comes from Green's function $\left\langle\left\langle S^{+} S^{-} \mid S^{+} S^{-}\right\rangle\right\rangle$; Green's function $\left\langle\left\langle S^{+} S^{-} \mid S^{z} S^{z}\right\rangle\right\rangle,\left\langle\left\langle S^{z} S^{z} \mid S^{+} S^{-}\right\rangle\right\rangle$gives contribution only to the diagonal components of the tensor. The nondiagonal components of the tensor have the terms which are proportional to $\sin \left(k_{x} / 2\right) \sin \left(k_{y} / 2\right)$; these terms lead to the appearance of the peak at the edge of the Brillouin zone [1].

The results obtained describe the two-magnon scattering and are similar to those obtained for antiferromagnetics [1] but the condition of homeopolarity is not valid for the $t-J$ model and so the hole doping level is not equal to zero. It has led in our approximation to the appearance of the factor $(1-\delta)^{4}$ in the expression for the scattering tensor. In general, the influence of the doping on the scattering at the hole concentration increase should be more complicated due to a rapid destruction of the antiferromagnetic state. The changes in the scattering spectrum in this case can be investigated even based on the expression (32) at the proper consideration of the hole-magnon scattering.

\section{General case}

Let us return now to the Hubbard model and consider the consequences of introducing the excited atomic states [9] into this model. Into the Hamiltonian (7) we insert the additional terms which are similar to the ones used in the case of $t-J$ model:

$$
\begin{aligned}
\hat{H}= & \sum_{i, j} t_{i, j} \hat{c}_{i, \sigma}^{\dagger} \hat{c}_{j, \sigma}+U \sum_{i} \hat{n}_{i, \uparrow} \hat{n}_{i, \downarrow}-\sum_{i} \mu \hat{n}_{i} \\
& +(E-\mu) \sum_{i, \nu} \hat{a}_{\nu i, \sigma}^{\dagger} \hat{a}_{\nu i, \sigma}+\sum_{i, \nu} M_{i j}^{\lambda \nu} \hat{a}_{\lambda j, \sigma^{\prime}}^{\dagger} \hat{a}_{\nu i, \sigma^{\prime}} \hat{c}_{i, \sigma^{\prime}}^{\dagger} \hat{c}_{j, \sigma^{\prime}}
\end{aligned}
$$

Here the operators $c, n$ refer to the ground state, the operators $a_{\lambda}, n_{\lambda}$ refer to the excited states. We do not include the term connected with the interaction $K_{i j}^{\alpha \beta}$ because it does not lead to the new contributions to the scattering tensor in comparison with that obtained from the term connected with the interaction $M_{i j}^{\alpha \beta}$. The dipole momentum is taken in the form:

$$
P_{i}^{\alpha}=e R_{i}^{\alpha}\left[n_{\uparrow i}+n_{\downarrow i}\right]+\sum_{\lambda}\left[n_{\lambda \uparrow i}+n_{\lambda \downarrow i}\right]+d^{\alpha} \sum_{\sigma}\left[a_{\alpha i \sigma}^{+} c_{i \sigma}+\text { h.c. }\right]
$$

which takes into account both types of the contributions considered above. 
Similar to the previous cases we can obtain the following expression for the operator Green's function $\left\{\left\{M^{\alpha} \mid M^{\beta}\right\}\right\}$ :

$$
\begin{aligned}
&\left\{\left\{M_{k}^{\alpha} \mid M_{l}^{\beta}\right\}\right\}=\hbar \frac{e^{2} d^{\alpha} d^{\beta}}{2 \pi} \frac{1}{\left(\hbar \omega_{1}-E\right)\left(\hbar \omega_{2}-E\right)} \delta_{\alpha, \beta} \\
& \times\left[-\sum_{i} \delta_{k, l} t_{i, k}\left(X_{i}^{31} X_{k}^{13}+X_{i}^{41} X_{k}^{14}\right)\right. \\
&\left.-M_{l, k}\left(X_{k}^{33} X_{l}^{33}+X_{k}^{44} X_{l}^{44}+X_{k}^{43} X_{l}^{34}+X_{k}^{34} X_{l}^{43}\right)\right] \\
&+ \frac{\hbar e^{2} R_{k}^{\alpha} R_{l}^{\beta}}{2 \pi \hbar^{2} \omega_{1} \omega_{2}} \sum_{i, j, s} t_{i, j} t_{s, j}\left(\delta_{i, k}-\delta_{j, k}\right)\left(\delta_{j, l}-\delta_{s, l}\right) \\
& \times\left(X_{i}^{31} X_{j}^{44} X_{\mathrm{s}}^{13}+X_{i}^{41} X_{j}^{33} X_{\mathrm{s}}^{14}-X_{i}^{41} X_{j}^{34} X_{\mathrm{s}}^{13}-X_{i}^{31} X_{j}^{43} X_{\mathrm{s}}^{14}\right) \\
&- \hbar \frac{e^{2} d^{\alpha} d^{\beta}}{2 \pi} \sum_{i j}\left[\frac{1}{\left(\hbar \omega_{1}-E+U\right)\left(\hbar \omega_{2}-E\right)\left(\hbar \omega_{2}-E\right)}\right. \\
&\left.-\frac{1}{\left(\hbar \omega_{1}+E-U\right)\left(\hbar \omega_{1}+E\right)\left(\hbar \omega_{2}+E\right)}\right] \delta_{k l} t_{i k} t_{j l} \\
& \times\left(X_{i}^{31} X_{j}^{14} X_{k}^{43}+X_{i}^{41} X_{j}^{13} X_{k}^{34}-X_{i}^{41} X_{j}^{14} X_{k}^{33}-X_{i}^{31} X_{j}^{13} X_{k}^{44}\right) \\
&- 2 \hbar \frac{e^{2} d^{\alpha} d^{\beta}}{2 \pi} \sum_{i j}\left[\frac{1}{\left(\hbar \omega_{1}-E+U\right)\left(\hbar \omega_{2}-E\right)\left(\hbar \omega_{2}-E+U\right)}\right. \\
&\left.+\frac{1}{\left(\hbar \omega_{1}+E-U\right)\left(\hbar \omega_{2}+E-U\right)\left(\hbar \omega_{1}+E\right)}\right] \delta_{k l} t_{i k} t_{j l} \\
& \times\left(X_{i}^{31} X_{j}^{14} X_{k}^{43}+X_{i}^{41} X_{j}^{13} X_{k}^{34}-X_{i}^{41} X_{j}^{14} X_{k}^{33}-X_{i}^{31} X_{j}^{13} X_{k}^{44}\right)(39
\end{aligned}
$$

(only resonant contributions are presented). One can see the presence of the resonant terms with the frequencies:

$$
\hbar \omega_{1} \sim E ; \quad \hbar \omega_{1} \sim U ; \quad \hbar \omega_{1} \sim E-U ; \quad \hbar \omega_{1} \sim U-E .
$$

The formula (39) for the function $\left\{\left\{M^{\alpha} \mid M^{\beta}\right\}\right\}$ includes new terms in comparison with the expression (12). One of them (proportional to the interaction constant $M_{k, l}$ ) has the structure of the scalar product $\vec{S}_{l} \vec{S}_{k}$. Similar to (25) it leads to the magnon scattering. A similar contribution can be obtained from the resonant term proportional to $\left(\hbar \omega_{1}-U\right)^{-1}$ in the case $i=j$. Considering such terms and maintaining only the resonant contributions (with factors $\left(\hbar \omega_{1}-U\right)^{-1}$ and $\left.\left(\hbar \omega_{1,2}-E\right)^{-1}\right)$ we can write the formula for the diagonal components of the magnon scattering tensor as follows:

$$
\begin{aligned}
H^{\alpha \alpha, \alpha \alpha(\beta \beta, \beta \beta)}\left(\omega_{1}, \omega_{2}\right)= & \frac{(1-\delta)^{4} 2 \pi \mathrm{e}^{4} \hbar^{2}}{\left(\mathrm{e}^{\beta \hbar \omega}-1\right)} \sum_{k}\left(v_{k}^{2}+u_{k}^{2}\right)^{2} \delta\left(\omega-2 \omega_{k}\right) \\
\times & \left\{\cos ^{2}\left(k_{y} / 2\right) \cos ^{2}\left(k_{x} / 2\right)\left(2 n_{k}+1\right)\right. \\
& \times\left[\frac{4 d^{x 2} M^{x x}}{\left(\hbar \omega_{1}-E\right)\left(\hbar \omega_{2}-E\right)}+\frac{2 a^{2} t^{2}}{\hbar \omega_{1} \hbar \omega_{2}\left(\hbar \omega_{1}-U\right)}\right]^{2} \\
& +\sin ^{2}\left(k_{y} / 2\right) \sin ^{2}\left(k_{x} / 2\right) \sin ^{2}(2 \gamma)\left(2 n_{k}+1\right)
\end{aligned}
$$




$$
\left.\times\left[\frac{4 d^{x 2} M^{x y}}{\left(\hbar \omega_{1}-E\right)\left(\hbar \omega_{2}-E\right)}+\frac{2 a^{2} t^{2}}{\hbar \omega_{1} \hbar \omega_{2}\left(\hbar \omega_{1}-U\right)}\right]^{2}\right\}
$$

(magnon Green's functions were calculated here similar to (34)). The components $H^{\alpha \alpha, \beta \beta}, H^{\beta \beta, \alpha \alpha}$ differ from $H^{\alpha \alpha, \alpha \alpha}$ only by the sign of the second term. The nondiagonal components can be written as follows:

$$
\begin{aligned}
& H^{\alpha \beta, \alpha \beta(\beta \alpha, \beta \alpha)}\left(\omega_{1}, \omega_{2}\right)=2 \pi \frac{e^{4} \hbar^{2}}{\left(\mathrm{e}^{\beta \hbar \omega}-1\right)} \sum_{k}\left(v_{k}^{2}+u_{k}^{2}\right)^{2} \\
& \quad \times \delta\left(\omega-2 \omega_{k}\right)\left[\frac{4 d^{x 2} M^{x y}}{\left(\hbar \omega_{1}-E\right)\left(\hbar \omega_{2}-E\right)}+\frac{2 a^{2} t^{2}}{\hbar \omega_{1} \hbar \omega_{2}\left(\hbar \omega_{1}-U\right)}\right]^{2} \\
& \quad \times\left(2 n_{k}+1\right) \cos ^{2}(2 \gamma) \sin ^{2}\left(k_{y} / 2\right) \sin ^{2}\left(k_{x} / 2\right)(1-\delta)^{4} .
\end{aligned}
$$

Here the case is considered, when the directions of light polarization do not coincide with the crystallographic axes; $\gamma$ is the corresponding angle $(\gamma=0$ in the case considered in the previous section). We can see that if $\gamma \neq 0$, the diagonal components of the tensor $H^{\alpha \alpha, \alpha \alpha(\beta \beta, \beta \beta)}\left(\omega_{1}, \omega_{2}\right)$ also lead to the appearance of the peak at the edge of the Brillouin zone [1]. The factor $(1-\delta)^{4}$ is present in the expression for the scattering tensor contrary to the results obtained in [1]. This factor gives a rough estimate of the hole effect on the scattering (in [1] the case of pure antiferromagnets was considered).

\section{The pseudospin-electron model}

In this section we consider the main contributions to Raman scattering in a more complicated case of the pseudospin-electron model. This model can be considered as an extension of the Hubbard model by the inclusion of the interaction with pseudospin degrees of freedom. The Hamiltonian of the model has the following form $[8]$ :

$$
H=\sum_{i} H_{i}+\sum_{i, j, \sigma} t_{i, j} \hat{c}_{i, \sigma}^{\dagger} \hat{c}_{j, \sigma}
$$

here the Hamiltonian $H_{i}$ describes (besides the Hubbard electron correlation) the interaction with the local anharmonic vibrational modes, described by pseudospins:

$$
H_{i}=U n_{i \uparrow} n_{i \downarrow}-\mu\left(n_{i \uparrow}+n_{i \downarrow}\right)+g\left(n_{i \uparrow}+n_{i \downarrow}\right) S_{i}^{z}-\Omega S_{i}^{x}-h S_{i}^{z}
$$

New terms in $H_{i}$ have the following meaning: interaction with the local vibrations ( $g$-term), splitting of the vibrational mode by tunnelling ( $\Omega$-term), asymmetry of the vibrational mode ( $h$-term).

In the case of narrow electron bands $(t \ll U)$, the single-site Hamiltonian $H_{i}$ plays the role of a zero order approximation. Therefore let us introduce the following single-site basis of states $\left|n_{i \uparrow}, n_{i \downarrow}, S_{i}^{z}\right\rangle[8]$ :

$$
\begin{aligned}
|1\rangle=\left|0,0, \frac{1}{2}\right\rangle, & |\widetilde{1}\rangle=\left|0,0,-\frac{1}{2}\right\rangle, & |2\rangle=\left|1,1, \frac{1}{2}\right\rangle, & |\widetilde{2}\rangle=\left|1,1,-\frac{1}{2}\right\rangle, \\
|3\rangle=\left|0,1, \frac{1}{2}\right\rangle, & |\widetilde{3}\rangle=\left|0,1,-\frac{1}{2}\right\rangle, & |4\rangle=\left|1,0, \frac{1}{2}\right\rangle, & |\widetilde{4}\rangle=\left|1,0,-\frac{1}{2}\right\rangle .
\end{aligned}
$$


It is useful to use the Hubbard operators $X^{R S}=|R\rangle\langle S|$, acting in the space spanned by the vectors (45). The Hamiltonian can be reduced to a diagonal form, using the transformation

$$
|R\rangle=\cos \left(\phi_{r}\right)|r\rangle+\sin \left(\phi_{r}\right)|\widetilde{r}\rangle, \quad|\widetilde{R}\rangle=\cos \left(\phi_{r}\right)|\widetilde{r}\rangle-\sin \left(\phi_{r}\right)|r\rangle,
$$

where

$$
\begin{aligned}
& \cos \left(2 \phi_{1}\right)=\frac{-h}{\sqrt{h^{2}+\Omega^{2}}}, \quad \cos \left(2 \phi_{2}\right)=\frac{2 g-h}{\sqrt{(2 g-h)^{2}+\Omega^{2}}} \\
& \cos \left(2 \phi_{3}\right)=\cos \left(2 \phi_{4}\right)=\frac{g-h}{\sqrt{(g-h)^{2}+\Omega^{2}}}
\end{aligned}
$$

Thus we get for $H_{i}$ in terms of the operators $X^{r s}$ :

$$
H_{i}=\sum_{r} \varepsilon_{r} X_{i}^{r r}
$$

where

$$
\begin{aligned}
& \varepsilon_{1, \widetilde{1}}= \pm \frac{1}{2} \sqrt{h^{2}+\Omega^{2}}, \quad \varepsilon_{2, \widetilde{2}}=2 E_{0}+U \pm \frac{1}{2} \sqrt{(2 g-h)^{2}+\Omega^{2}}, \\
& \varepsilon_{3, \widetilde{3}}=\varepsilon_{3, \widetilde{3}}=E_{0} \pm \frac{1}{2} \sqrt{(g-h)^{2}+\Omega^{2}}
\end{aligned}
$$

The total Hamiltonian is given by the expression

$$
H=\sum_{i, r} \varepsilon_{r} X_{i}^{r r}+\sum_{i, j, \sigma} t_{i, j} \hat{c}_{i, \sigma}^{\dagger} \hat{c}_{j, \sigma}
$$

with

$$
\begin{aligned}
\hat{c}_{i, \uparrow}^{\dagger}= & \cos \left(\phi_{4}-\phi_{1}\right)\left(X_{i}^{41}+X_{i}^{\widetilde{41}}\right)-\sin \left(\phi_{4}-\phi_{1}\right)\left(X_{i}^{4 \widetilde{1}}-X_{i}^{\widetilde{4} 1}\right) \\
& +\cos \left(\phi_{2}-\phi_{3}\right)\left(X_{i}^{23}+X_{i}^{\widetilde{23}}\right)-\sin \left(\phi_{2}-\phi_{3}\right)\left(X_{i}^{2 \widetilde{3}}-X_{i}^{\widetilde{23}}\right), \\
\hat{c}_{i, \downarrow}^{\dagger}= & \cos \left(\phi_{3}-\phi_{1}\right)\left(X_{i}^{31}+X_{i}^{\widetilde{31}}\right)-\sin \left(\phi_{4}-\phi_{1}\right)\left(X_{i}^{3 \widetilde{1}}-X_{i}^{\widetilde{3} 1}\right) \\
& -\cos \left(\phi_{2}-\phi_{4}\right)\left(X_{i}^{24}+X_{i}^{\widetilde{24}}\right)+\sin \left(\phi_{2}-\phi_{4}\right)\left(X_{i}^{2 \widetilde{4}}-X_{i}^{\widetilde{24}}\right) .
\end{aligned}
$$

Let us choose the dipole momentum of a unit cell in the form

$$
M_{i}^{\alpha}=d_{\mathrm{e}}\left(n_{\uparrow, i}+n_{\downarrow, i}\right)+d_{\mathrm{s}} S_{i}^{z},
$$

or in terms of the operators $X^{r s}$

$$
\begin{aligned}
M_{i}^{\alpha}= & d_{\mathrm{e}}\left(X_{i}^{22}+X_{i}^{\widetilde{22}}-X_{i}^{11}-X_{i}^{\widetilde{11}}+1\right) \\
& +d_{\mathrm{s}} \sum_{r}\left[\cos \left(2 \phi_{r}\right)\left(X_{i}^{r r}-X_{i}^{\widetilde{r r}}\right)+\sin \left(2 \phi_{r}\right)\left(X_{i}^{r \widetilde{r}}+X_{i}^{\widetilde{r r}}\right)\right],
\end{aligned}
$$

The expression (52) was used in [8] in the case of quasi-two-dimensional crystal structure of the $\mathrm{YBa}_{2} \mathrm{Cu}_{3} \mathrm{O}_{7-\delta}$ type systems and corresponds to the "transverse" 
component of polarization vector. Two equilibrium positions of anharmonic ion described by the pseudospin values $S_{i}^{z}= \pm \frac{1}{2}$ are oriented normally to the conducting $(a, b)$ plane. The first term in (52) is connected as the (8) term with the nonhomeopolarity of filling of the electron states on the site; the second term has an ionic origin and is determined by the particle localization (that is, the orientation of pseudospin). To calculate the unaveraged Green's function $\left\{\left\{M_{k}^{\alpha} \mid M_{l}^{\beta}\right\}\right\}$ we make an operator expansion, using $t$ as a formal small parameter [12]. At first we use the equation of motion, which is presented in the form (5):

$$
\begin{aligned}
\left\{\left\{M_{k}^{\alpha} \mid M_{l}^{\beta}\right\}\right\}= & \frac{\delta_{k, l} d_{\mathrm{s}}^{2}}{\hbar \omega_{1}} \sum_{r} \sin \left(4 \phi_{r}\right)\left(X^{r \widetilde{r}}-X^{\widetilde{r r}}\right) \\
& +\sum_{r} \frac{\delta_{k, l} d_{\mathrm{s}}^{2}}{\left(\hbar \omega_{1}-E_{r}+E_{\widetilde{r}}\right)}\left[\sin \left(4 \phi_{r}\right) X^{\widetilde{r r}}-\sin ^{2}\left(2 \phi_{r}\right)\left(X^{r r}-X^{\widetilde{r r}}\right)\right] \\
& -\sum_{r} \frac{\delta_{k, l} d_{\mathrm{s}}^{2}}{\left(\hbar \omega_{1}+E_{r}-E_{\widetilde{r}}\right)}\left[\sin \left(4 \phi_{r}\right) X^{r \widetilde{r}}-\sin ^{2}\left(2 \phi_{r}\right)\left(X^{r r}-X^{\widetilde{r r}}\right)\right] \\
& +\frac{d_{\mathrm{e}}}{\hbar \omega_{1}} \sum_{i, j, \sigma} t_{i, j}\left(\delta_{i, k}-\delta_{j, k}\right)\left\{\left\{\hat{c}_{i, \sigma}^{\dagger} \hat{c}_{j, \sigma} \mid \hat{M}_{l}^{\beta}\right\}\right\} \\
& +\sum_{r} \frac{d_{\mathrm{s}}}{\hbar \omega_{1}} \sum_{i, j, \sigma} t_{i, j} \cos \left(2 \phi_{r}\right)\left\{\left\{\left[X^{r r}-X^{\widetilde{r r}}, \hat{c}_{i, \sigma}^{\dagger} \hat{c}_{j, \sigma}\right] \mid \hat{M}_{l}^{\beta}\right\}\right\} \\
& +\sum_{r} \frac{d_{\mathrm{s}}}{\left(\hbar \omega_{1}-E_{r}+E_{\widetilde{r}}\right)} \sum_{i, j, \sigma} t_{i, j} \sin \left(2 \phi_{r}\right)\left\{\left\{\left[X^{\widetilde{r r}}, \hat{c}_{i, \sigma}^{\dagger} \hat{c}_{j, \sigma}\right] \mid \hat{M}_{l}^{\beta}\right\}\right\} \\
& +\sum_{r} \frac{d_{\mathrm{s}}}{\left(\hbar \omega_{1}+E_{r}-E_{\widetilde{r}}\right)} \sum_{i, j, \sigma} t_{i, j} \sin \left(2 \phi_{r}\right)\left\{\left\{\left[X^{r \widetilde{r}}, \hat{c}_{i, \sigma}^{\dagger} \hat{c}_{j, \sigma}\right] \mid \hat{M}_{l}^{\beta}\right\}\right\} .
\end{aligned}
$$

We can see that contrary to the Hubbard and $t-J$ models, in the case of the pseudospin-electron model Green's function $\left\{\left\{M_{k}^{\alpha} \mid M_{l}^{\beta}\right\}\right\}$ possesses the terms, which are of the zero order with respect to the electron transfer parameter. The appearing of these terms is caused by the dipole momentum dynamics, connected with the pseudospin reorientation. The calculation of Green's functions $\left\langle\left\langle X^{r r} \mid X^{p p}\right\rangle\right\rangle,\left\langle\left\langle X^{r r} \mid X^{\widetilde{p p}}\right\rangle\right\rangle$, $\left\langle\left\langle X^{r r} \mid X^{p \widetilde{p}}\right\rangle\right\rangle$ etc. and the investigation, on this basis, of corresponding contributions to the Raman scattering tensor was performed in [13]. The frequency dependence of scattering at various values of electron concentration and temperature was considered.

Now for the function (54) we use the equation of motion in the form (6). The expression obtained after this procedure is very cumbersome, so here we write out only one term thereof:

$$
\begin{aligned}
& \sum_{i, j, \sigma} t_{i, j}\left(\delta_{i, k}-\delta_{j, k}\right)\left\{\left\{\hat{c}_{i, \sigma}^{\dagger} \hat{c}_{j, \sigma} \mid\left(n_{\uparrow l}+n_{\downarrow l}\right)\right\}\right\}= \\
& =\frac{1}{\hbar \omega_{2}} \sum_{\substack{i, j, \sigma \\
s, p, \sigma^{\prime}}} t_{i, j}\left(\delta_{i, k}-\delta_{j, k}\right) t_{s, p}\left(\delta_{p, l}-\delta_{s, l}\right)\left\{\left\{\hat{c}_{i, \sigma}^{\dagger} \hat{c}_{j, \sigma} \mid \hat{c}_{s, \sigma^{\prime}}^{\dagger} \hat{c}_{p, \sigma^{\prime}}\right\}\right\} \\
& \quad-\frac{1}{2 \pi \omega_{2}} \sum_{i, j, \sigma} t_{i, j}\left(\delta_{i, k}-\delta_{j, k}\right)\left(\delta_{i, l}-\delta_{j, l}\right) \hat{c}_{i, \sigma}^{\dagger} \hat{c}_{j, \sigma}
\end{aligned}
$$


The structure of the other terms is similar. Then we again write the equation of motion in the form (6) in order to find Green's function $\left\{\left\{\hat{c}_{i, \sigma}^{\dagger} \hat{c}_{j, \sigma} \mid \hat{c}_{s, \sigma^{\prime}}^{\dagger} \hat{c}_{p, \sigma^{\prime}}\right\}\right\}$ up to the terms of the second order in $t$. Considering the resonant term, which describes the one-electron transition $\widetilde{1} \widetilde{4} \rightarrow 14$, we have

$$
\begin{aligned}
& \sum_{\substack{i, j, s, p, \sigma^{\prime}}} t_{i, j}\left(\delta_{i, k}-\delta_{j, k}\right) t_{s, p}\left(\delta_{p, l}-\delta_{s, l}\right) \cos ^{2}\left(\phi_{4}-\phi_{1}\right)\left\{\left\{X_{i}^{\widetilde{41}} X_{j}^{14} \mid \hat{c}_{s, \sigma^{\prime}}^{\dagger} \hat{c}_{p, \sigma^{\prime}}\right\}\right\}= \\
& =\sum_{\substack{i, j, s, p}} \frac{t_{i, j}\left(\delta_{i, k}-\delta_{j, k}\right) t_{s, p}\left(\delta_{p, l}-\delta_{s, l}\right) \hbar \cos ^{2}\left(\phi_{4}-\phi_{1}\right)}{2 \pi\left(\hbar \omega_{1}-E_{4}+E_{1}+E_{\widetilde{4}}-E_{\widetilde{1}}\right)} \\
& \quad \times\left\{X_{i}^{\widetilde{41}}\left[\cos \left(\phi_{4}-\phi_{1}\right)\left(X_{j}^{11}+X_{j}^{44}\right)-\sin \left(\phi_{4}-\phi_{1}\right)\left(X_{j}^{1 \widetilde{1}}-X_{j}^{\widetilde{44}}\right)\right] \hat{c}_{s, \uparrow}\right. \\
& \quad-\hat{c}_{s, \uparrow}^{\dagger} X_{i}^{14}\left[\cos \left(\phi_{4}-\phi_{1}\right)\left(X_{j}^{\widetilde{11}}+X_{j}^{\widetilde{44}}\right)-\sin \left(\phi_{4}-\phi_{1}\right)\left(X_{j}^{\widetilde{44}}-X_{j}^{1 \widetilde{1}}\right)\right] \\
& \quad+X_{i}^{\widetilde{41}}\left[\cos \left(\phi_{4}-\phi_{1}\right) X_{j}^{34}+\sin \left(\phi_{4}-\phi_{1}\right) X_{j}^{\widetilde{3} 4}\right] \hat{c}_{s, \downarrow}- \\
& \left.\quad-\hat{c}_{s, \downarrow}^{\dagger} X_{i}^{14}\left[\cos \left(\phi_{4}-\phi_{1}\right) X_{j}^{\widetilde{43}}-\sin \left(\phi_{4}-\phi_{1}\right) X_{j}^{\widetilde{43}}\right]\right\} .
\end{aligned}
$$

We have omitted in this expression the terms, connected with the doubly occupied states $|2\rangle,|\widetilde{2}\rangle$. If $\mathrm{i}=\mathrm{s}$, the expression can be written as follows:

$$
\begin{aligned}
& \frac{\sum_{i, j} t_{i, j}^{2}\left(\delta_{i, k}-\delta_{j, k}\right)\left(\delta_{j, l}-\delta_{i, l}\right) \hbar \cos ^{2}\left(\phi_{4}-\phi_{1}\right)}{2 \pi\left(\hbar \omega_{1}-E_{4}+E_{1}+E_{\widetilde{4}}-E_{\widetilde{1}}\right)} \\
& \times\left\{\cos ^{2}\left(\phi_{4}-\phi_{1}\right)\left[X_{i}^{11}\left(X_{j}^{\widetilde{44}}+X_{j}^{\widetilde{33}}\right)-X_{i}^{\widetilde{11}}\left(X_{j}^{44}+X_{j}^{33}\right)\right]\right. \\
& \quad-\sin \left(\phi_{4}-\phi_{1}\right) \cos \left(\phi_{4}-\phi_{1}\right) \\
& \left.\quad \times\left[\left(X_{i}^{\widetilde{44}}+X_{i}^{\widetilde{3} 3}\right)\left(X_{j}^{11}+X_{j}^{\widetilde{11}}\right)+X_{i}^{\widetilde{11}}\left(X_{j}^{44}+X_{j}^{33}+X_{j}^{\widetilde{44}}+X_{j}^{\widetilde{33}}\right)\right]\right\} .
\end{aligned}
$$

One can see that the terms connected with the electron spin reorientation aren't present in this expression because the subband $\widetilde{1} \widetilde{4}$ is created by the pseudospin reorientation - for the Hubbard model this subband is not present. This expression includes the terms which effectively take into account the electron correlations on the neighbouring lattice sites in connection with the pseudospin dynamics. The corresponding Raman scattering contributions can be important in the presence of the charge ordered states with the modulation of the electron density and orientation of pseudospins (the possibility of such ordering in the pseudospin-electron model with the unit cell doubling was investigated in [14]).

Let us consider another term, which is resonant at the transition $\widetilde{1} \widetilde{4} \rightarrow \widetilde{3} \widetilde{2}$

$$
\begin{aligned}
& \sum_{\substack{i, j, s, p, \sigma^{\prime}}} t_{i, j}\left(\delta_{i, k}-\delta_{j, k}\right) t_{s, p}\left(\delta_{p, l}-\delta_{s, l}\right) \cos \left(\phi_{2}-\phi_{3}\right) \cos \left(\phi_{4}-\phi_{1}\right) \\
& \times\left\{\left\{X_{i}^{\widetilde{41}} X_{j}^{\widetilde{3} 2}-X_{i}^{\widetilde{31}} X_{j}^{\widetilde{42}} \mid \hat{c}_{s, \sigma^{\prime}}^{\dagger} \hat{c}_{p, \sigma^{\prime}}\right\}\right\}= \\
& \quad \frac{\sum_{i, j} \hbar \cos \left(\phi_{4}-\phi_{1}\right) \cos \left(\phi_{2}-\phi_{3}\right) t_{i, j}\left(\delta_{i, k}-\delta_{j, k}\right) t_{s, j}\left(\delta_{j, l}-\delta_{s, l}\right)}{2 \pi\left(\hbar \omega_{1}-E_{\widetilde{1}}+E_{\widetilde{4}}+E_{\widetilde{3}}-E_{\widetilde{2}}\right)} \\
& \quad \times\left\{X_{i}^{\widetilde{41}}\left[\cos \left(\phi_{2}-\phi_{3}\right) X_{j}^{\widetilde{33}}+\sin \left(\phi_{2}-\phi_{3}\right) X_{j}^{\widetilde{3}}\right] \hat{c}_{s, \uparrow}\right.
\end{aligned}
$$




$$
\begin{aligned}
& -X_{i}^{\widetilde{41}}\left[\cos \left(\phi_{2}-\phi_{3}\right) X_{j}^{\widetilde{34}}+\sin \left(\phi_{2}-\phi_{3}\right) X_{j}^{\widetilde{3} 4}\right] \hat{c}_{s, \downarrow} \\
& +X_{i}^{\widetilde{31}}\left[\cos \left(\phi_{2}-\phi_{3}\right) X_{j}^{\widetilde{44}}+\sin \left(\phi_{2}-\phi_{3}\right) X_{j}^{\widetilde{4} 4}\right] \hat{c}_{s, \downarrow} \\
& \left.-X_{i}^{\widetilde{31}}\left[\cos \left(\phi_{2}-\phi_{3}\right) X_{j}^{\widetilde{43}}+\sin \left(\phi_{2}-\phi_{3}\right) X_{j}^{\widetilde{4}}\right] \hat{c}_{s, \uparrow}\right\}
\end{aligned}
$$

having omitted the terms, including the states $|2\rangle,|\widetilde{2}\rangle$. Separating the terms with $i=s$, we obtain the following formula:

$$
\begin{aligned}
& \frac{\sum_{i, j} \hbar \cos \left(\phi_{4}-\phi_{1}\right) \cos \left(\phi_{2}-\phi_{3}\right) t_{i, j}^{2}\left(\delta_{i, k}-\delta_{j, k}\right)\left(\delta_{j, l}-\delta_{i, l}\right)}{2 \pi\left(\hbar \omega_{1}-E_{\widetilde{1}}+E_{\widetilde{4}}+E_{\widetilde{3}}-E_{\widetilde{2}}\right)} \\
& \times\left\{\cos \left(\phi_{2}-\phi_{3}\right) \cos \left(\phi_{4}-\phi_{1}\right)\left[X_{i}^{\widetilde{44}} X_{j}^{\widetilde{33}}+X_{i}^{\widetilde{33}} X_{j}^{\widetilde{44}}-X_{i}^{\widetilde{43}} X_{j}^{\widetilde{34}}-X_{i}^{\widetilde{34}} \widetilde{X_{j}^{43}}\right]\right. \\
& \quad+\sin \left(\phi_{2}-\phi_{3}\right) \sin \left(\phi_{4}-\phi_{1}\right)\left[X_{i}^{\widetilde{43}} X_{j}^{\widetilde{3} 4}+X_{i}^{\widetilde{34}} X_{j}^{\widetilde{43}}-X_{i}^{\widetilde{4} 4} X_{j}^{\widetilde{3} 3}-X_{i}^{\widetilde{33}} X_{j}^{\widetilde{44}}\right] \\
& \left.\quad+\sin \left(2 \phi_{4}-\phi_{2}-\phi_{1}\right)\left[X_{i}^{\widetilde{43}} X_{j}^{\widetilde{34}}+X_{i}^{\widetilde{43}} X_{j}^{\widetilde{34}}-X_{i}^{\widetilde{44}} X_{j}^{\widetilde{33}}-X_{i}^{\widetilde{44}} X_{j}^{\widetilde{33}}\right]\right\} .
\end{aligned}
$$

In this expression there are both the terms, connected with the reorientation of the electron spins and the ones, connected with the reorientation of the pseudospins. Therefore Raman scattering at the transitions between the band separated by $U$ is connected with the electron spin dynamics, very similar to that described by Fleury and Loudon approach [1] (creation of magnon pairs), as well as with the pseudospin dynamics. As it was shown in [8], the interaction with pseudospins leads to the modulation of the parameter of the effective exchange interaction, depending on the state of the pseudospin subsystem. The role of this effect can be studied by calculating the Green's functions $\left\langle\left\langle X^{\widetilde{r r}} X^{\widetilde{p p}} \mid X^{\widetilde{s s}} X^{\widetilde{q q}}\right\rangle\right\rangle,\left\langle\left\langle X^{\widetilde{43}} X^{\widetilde{34}} \mid X^{\widetilde{43}} X^{\widetilde{34}}\right\rangle\right\rangle$ and others, and by investigating, on this basis, the expression for the Raman scattering tensor.

\section{Conclusions}

The method of constructing the polarizability operator for systems with a strong short-range correlation between electrons is developed in this work. The operator expansions in powers of the parameter of the electron hopping are carried out to calculate the polarizability operator. The expressions for the Raman scattering tensor in terms of the correlation functions calculated on the spin operators or Hubbard operators are obtained for the Hubbard, $t-J$ and pseudospin-electron models. It is shown that for the pure Hubbard model, Raman scattering in the doped case cannot be reduced to the nonresonant scattering only. The resonant contributions are present as well having the character of the two-electron transitions in the conductance band. In the case of the $t-J$ model, the scattering due to the magnon pair creation is modified by the magnon-hole scattering process. In the simplest approximation the scattering intensity decreases in the doped case proportionally to the factor $(1-\delta)^{4}$, where $\delta$ is the concentration of holes. It is established that the inclusion of the excited electron states (and the corresponding exchange interactions) leads in the Hubbard model to the appearance, in the explicit form, of the 
scattering components which correspond to the Fleury-Loudon [1] mechanism of the two-magnon scattering. In general, two different contributions to the polarizability operator, one of which is connected with the nonhomeopolarity of filling of the electron states on a site and another is responsible for the dipole transitions to the excited states, lead to similar contributions to the scattering tensor.

In the case of the pseudospin-electron model the polarizability operator is not equal to zero if the electron hopping isn't present. This corresponds to the pure pseudospin scattering that is caused by the dipole transitions connected with the reorientation of pseudospins. Analysing the expression for the polarizability operator, we can see that there exist resonant transitions between the electron energy subbands which appear due to the pseudospin-electron interaction. Investigating the terms which are proportional to $t^{2}$ we have shown that the two main scattering mechanisms can be separated between others. The first one is connected with the correlation of the pseudospin dynamics with electron filling on the neighbouring sites, which can be important in the case of the charge and pseudospin spatial modulation. The second one is analogous to the two-magnon scattering in antiferromagnets which is here accompanied by the reorientation of pseudospins.

\section{References}

1. Fleury P., Loudon R. Scattering of light by one- and two-magnon excitations. // Phys. Rev., 1968, vol. 166, No. 2, p. 514-530.

2. Shastry B.S., Shraiman B.I. Theory of Raman scattering in Mott-Hubbard systems. // Phys. Rev. Lett., 1990, vol. 65, No. 8, p. 1068-1071.

3. Shastry B.S., Shraiman B.I. Raman scattering in Mott-Hubbard systems. // Int. J. Mod. Phys. B, 1991, vol. 5, No. 1\&2, p. 365-388

4. Cowley R.A. The lattice dynamics of an anharmonic crystal. // Advances. Phys., 1963, vol. 12 , No. 48, p. 421-481.

5. Barry R., Sharpe I.W. Raman scattering from impurities in semiconductors. I. General results. // Can. J. Phys., 1978, vol. 56, p. 550-559.

6. Stasyuk I.V., Ivankiv Ya.L. Raman scattering in crystals with ordering structure units. Preprint of the Institute for Theoretical Physics, ITP-87-57P, Kyiv, 1987, 25 p. (in Russian).

7. Stasyuk I.V., Shvaika A.M. On the electron spectrum of the Hubbard model including interactions with local anharmonicity vibrations. // Physica C, 1993, vol. 213, p. 5770 .

8. Stasyuk I.V., Shvaika A.M. Dielectric properties and electron spectrum of the Müller model in the HTSC theory. // Acta Physica Polonica, 1993, vol. 84, p. 293-313.

9. Stasyuk I.V., Mysakovych T.S. Raman scattering tensor for Hubbard and $t-J$ models. Preprint of the Institute for Condensed Matter Physics, ICMP-98-28E, Lviv, 1998, $12 \mathrm{p}$.

10. Plakida N.M., Oudovenko V.S., Yushankhai V.Yu. Temperature and doping dependence of the quasiparticle spectrum for holes in the spin-polaron model of copper oxides. // Phys. Rev. B, 1994, vol. 50, No. 9, p. 6431-6441.

11. Martinez G., Horsch P. Spin-polarons in the $t-J$ model. // Phys. Rev. B, 1991, vol. 44 , p. 317. 
12. Stasyuk I.V., Mysakovych T.S. Polarizability operator for pseudospin-electron model. Preprint of the Institute for Condenced Matter Physics, ICMP-98-34E, Lviv, 1998, 8 p.

13. Stasyuk I.V., Mysakovych T.S. Raman scattering in pseudospin-electron model. // J. Phys. Studies, 1999, vol. 3, No. 3, p. 344-358.

14. Stasyuk I.V., Shvaika A.M., Danyliv O.D. Dielectric instability and charge ordering in the local anharmonic model of high- $T_{\mathrm{c}}$ superconductors. // Molecular Phys. Reports, 1995, No. 9, p. 61-75.

\title{
Комбінаційне розсіяння світла в системах 3 сильною короткодіючою взаємодією
}

\author{
І.В.Стасюк, Т.С.Мисакович \\ Інститут фізики конденсованих систем НАН України, \\ 79011 Львів, вул. Свєнціцького, 1 \\ Отримано 29 вересня 1999 р.
}

Досліджено внески різного типу в комбінаційне розсіяння світла для моделі Хаббарда, $t-J$ та псевдоспін-електронної моделей. Для побудови оператора поляризованості використовується мікроскопічний підхід, здійснюючи операторні розклади в термінах операторів Хаббарда і використовуючи $t$ та $J$ як формальні параметри розкладу. До розгляду приймалися два різні внески до дипольного моменту: один пов'язаний з негомеополярністю заповнення електронних станів на вузлах гратки, інший - із дипольними переходами з основного у збуджені стани ( для випадку моделі Хаббарда) та із дипольним моментом псевдоспінів (для псевдоспін-електронної моделі). Отримано загальні вирази для компонент тензора розсіяння, які описують магнонне, електронне (внутрі- та міжзонне) та псевдоспінове розсіяння. Виділено резонансні та нерезонансні внески, вивчається їх роль при зміні концентрації дірок внаслідок легування. Досліджено вигляд тензора розсіяння в залежності від співвідношень між поляризацією падаючого і розсіяного світла.

Ключові слова: комбінаційне розсіяння світла, хаббардівська кореляція, псевдоспін-електронна модель, оператор поляризованості

PACS: 72.10.Dp, 72.10.Di, 74.20 